\title{
Dedifferentiated liposarcoma: when eribulin can make the difference
}

\author{
Andrea Sbrana*,1,2, Federico Paolieri ${ }^{1,2}$, Francesco Bloise ${ }^{1,2}$, Simona Manacorda ${ }^{1,2}$, \\ Amedeo Nuzzo ${ }^{1,2}$, Enrico Sammarco ${ }^{1,2}$, Luca Galli ${ }^{1,2}$ \& Alfredo Falcone ${ }^{1,2}$ \\ ${ }^{1}$ Medical Oncology Unit 2, Azienda Ospedaliero-Universitaria Pisana, Pisa, Italy \\ ${ }^{2}$ Department of Translational Research \& New Technologies in Medicine \& Surgery, University of Pisa, Pisa, Italy \\ *Author for correspondence: Tel..: 39 050992466; andreasbrana89@gmail.com
}

We describe the case of a male subject affected by retroperitoneal advanced, anthracycline-pretreated liposarcoma, who experienced a long, beneficial clinical effect from eribulin treatment. In March 2013, a left, paraortic, retroperitoneal mass was surgically removed and diagnosed as Mdm2-positive dedifferentiated liposarcoma. In June 2015, a CT scan revealed disease progression and first-line epirubicin/ifosfamide treatment was started, followed by epirubicin in monotherapy. In January 2017, following a new disease progression, the patient started a second-line eribulin treatment that went on for about 1 year with no major adverse events. The CT scans performed every 3-4 months showed stable disease. After 13 months of treatment, a CT scan revealed disease progression and 10 days later, the patient died of bowel perforation and peritonitis.

First draft submitted: 24 September 2019; Accepted for publication: 19 November 2019; Published online: 24 December 2019

Keywords: dedifferentiated $\bullet$ epirubicin $\bullet$ eribulin $\bullet$ ifosfamide $\bullet$ liposarcoma $\bullet$ Mdm2

Liposarcoma, a tumor of lipoblasts, is a rare mesenchymal neoplasm that affects deep soft tissues, involving retroperitoneum and popliteal fossa [1]. The frequency of liposarcoma at different sites is dependent on tumor subtypes. Dedifferentiated liposarcoma is much more common in retroperitoneal location [2]. Liposarcoma is divided into three subtypes: well-differentiated and dedifferentiated liposarcoma (WDLs/DDLs), myxoid and round cell liposarcoma and pleomorphic liposarcoma [3]. DDLs, along with well-differentiated liposarcoma/atypical lipomatous tumors, represent the largest group of liposarcomas; they are typically found in adult or elderly patients and they primarily affect the retroperitoneum or the limbs [4].

Liposarcomas are often found in a significantly advanced condition, due to the absence of distinctive symptoms or helpful biomarkers, representing a relevant problem in terms of clinical management. Surgery represents the elective therapy for liposarcomas, but the tendency to relapse, especially locally, is high and the disease often evolves to tumors that are not surgically amenable because of their extension or anatomical relationships to neighboring structures [5]. Unlike solid tumors, liposarcoma is not generally acknowledged to be responsive to chemotherapy. Therefore, the choice of an appropriate treatment and a follow-up system are necessary in order to improve the prognosis of patients with liposarcoma [6]. There is evidence that histological subtypes of sarcoma respond differently to distinct regimens of chemotherapy [7]. Advanced or metastatic DDLs can benefit from chemotherapy that can lead to partial responses or disease stabilization for variable periods, if not to complete disease remission [8]. Currently, doxorubicin, ifosfamide, gemcitabine, docetaxel, trabectedin and eribulin are included among the effective molecules in the treatment of liposarcoma [9]. Doxorubicin is the first-line treatment for advanced inoperable and metastatic disease, while other treatments are effective as second- and third-line options $[10,11]$.

Eribulin mesylate (eribulin) represents a novel relevant therapeutic tool for patients affected by advanced liposarcoma that has already progressed on previous anthracycline-based chemotherapy. Eribulin is a synthetic, microtubule-destabilizing agent, reproducing the cytotoxic part (macrocyclic lactone C1-C38) of halichondrin B, a natural compound isolated from the marine sponge Halichondria okadai [12]. Eribulin inhibits the microtubule dynamics, binding to the 'plus' ends of microtubules, irreversibly blocking their polymerization, and to soluble

Future Medicine 
tubulin, forming nonproductive tubulin aggregates that stop the spindle formation and alter their morphology [13]. In addition, eribulin can reverse epithelial to mesenchymal transition, enhance tumor blood perfusion and induce cell differentiation changes with up-regulation of differentiation markers [14].

Clinical trials have been finalized in breast cancer, soft tissue sarcomas and non-small-cell lung cancer [15-17].

In a Phase III clinical trial, eribulin, compared with dacarbazine, offered a statistically significant advantage in terms of overall survival ( 13.5 vs 11.5 months, hazard ratio $[\mathrm{HR}]=0.77 ; 95 \% \mathrm{CI}$ : $0.62-0.95 ; \mathrm{p}=0.0169)$. It was characterized by a very manageable toxicity profile, offering a very good tolerance even in fragile patients [18].

Eribulin is the first drug approved for patients with advanced liposarcoma demonstrating an improvement in overall survival.

In this clinical report, we describe the case of a patient affected by retroperitoneal advanced, anthracyclinepretreated liposarcoma who experienced a long, beneficial clinical effect following eribulin treatment.

\section{Case presentation}

We report the case of a 57-year-old male subject, married, with two children, a sales representative with a very active life (he used to play soccer with his friends every weekend). The patient's medical history was positive for a primary idiopathic neutropenia, but the patient had no history of important infective complications.

From April 2012 to March 2013, the patient experienced a significant weight loss (about $20 \mathrm{~kg}$ ), without any apparent appetite loss.

In March 2013, his general practitioner noticed a palpable mass in the right hypochondrium and addressed the patient to the local emergency room, where he underwent a CT scan that revealed a $15-\mathrm{cm}$ left, paraortic, retroperitoneal mass.

On 8 April 2013, the patient was admitted to the general surgery unit, where he underwent a surgical procedure to remove the retroperitoneal mass together with the left kidney and the adrenal gland. The histological examination was positive for a multifocal well-differentiated liposarcoma (with the major lesion measuring $18 \mathrm{~cm}$ in its maximum diameter), localized in the retroperitoneum, the right iliac fossa, back and along the right iliac vessels, along the right common iliac vessels, at the mesenteric root, along the internal spermatic vessels and in front of the urinary bladder. The kidney, the ureter and the renal vein were free of neoplasia, though liposarcoma cells were found in the periureteral tissue.

After the surgical procedure, the patient was referred to our medical oncology unit. We decided for a second histological examination in a dedicated pathology center, where the diagnosis was changed to Mdm2-positive dedifferentiated liposarcoma.

Since the surgical intervention was radical and the post-surgery CT scan was negative, we decided to start a strict follow-up program.

In June 2015, a new CT scan revealed a disease progression, with the appearance of a new lesion in the retroperitoneum, in strict relationship with the superior mesenteric vessels and the celiac trunk.

After a multidisciplinary meeting, when a surgical approach was excluded, we started a first-line treatment with epirubicin and ifosfamide. Because of the onset of severe neurotoxicity (drowsiness, mental confusion, disorientation in time and place) after the first cycle, we decided to keep on with epirubicin inmonotherapy, which was continued up to the maximum tolerable dose. CT scans in September and December 2015 revealed a stable disease.

In March 2016, a new CT scan revealed a minimal increase of the retroperitoneal lesions, but the patient refused to start a new cycle of therapy. Consequently, we opted for a strict follow-up schedule. In May and September 2016, the CT scans showed a stable disease.

In January 2017, a new CT scan revealed a disease progression, also involving the right urinary tract, and causing an increase in the creatinine level $(2.14 \mathrm{mg} / \mathrm{dl})$. The urologist programmed a nephrostomy placement that led to a progressive decrease of the creatinine levels. The patient also experienced the onset of intense abdominal pain (7 out of 10 in a Numerical Rating Scale), which was treated with oxycodone/naloxone 20/10 mg twice daily, respectively.

After the nephrostomy placement, the patient started a second-line treatment with eribulin $1.23 \mathrm{mg} / \mathrm{m}^{2}$ e.v. on days 1 and 8, in 21-day cycles. The treatment started in March 2017.

The therapy with eribulin went on for about 1 year with no major adverse event, with the exclusion of an episode of grade- 2 anemia at the beginning of the fourth cycle (hemoglobin $9.7 \mathrm{~g} / \mathrm{dl}$ ). The episode spontaneously resolved. The patient experienced no worsening of the neutrophil count and no febrile neutropenia or infective complications were observed throughout the entire period of treatment with eribulin. The CT scans performed every 3-4 months 
showed stable disease. After about 3 months of therapy, the abdominal pain gradually decreased and the patient was able to discontinue the opioid treatment 4 months after the beginning of eribulin administration.

In April 2018, after 13 months of treatment, the patient experienced the recurrence of abdominal pain (8 out of 10 in a Numerical Rating Scale), which was mainly localized in the upper abdominal quadrants. At the same time, we detected a new increase in the creatinine level $(4.13 \mathrm{mg} / \mathrm{dl})$. At the beginning of May 2018, a new CT scan confirmed a disease progression and 2 days later, the patient was admitted to the emergency room for bowel obstruction; 10 days later, the patient died because of bowel perforation and subsequent peritonitis.

\section{Conclusion}

This clinical case represents an example of a quite long-lasting clinical benefit derived from the use of eribulin in anthracycline-pretreated, dedifferentiated liposarcoma. Eribulin treatment allowed a significant extension of patient's survival with no negative impact on his quality of life, but rather with a positive discontinuation of major opioid therapy. Eribulin was demonstrated to be safe, even in presence of a personal history of idiopathic neutropenia, and no complications related to neutropenia or febrile neutropenia were observed.

In conclusion, eribulin is confirmed as a valid and safe therapeutic option for patients affected by advanced anthracycline-pretreated liposarcoma. Additional research and clinical observations in specific settings will be of major importance for a wider application of eribulin-based treatment regimens.

\section{Summary points}

- Liposarcoma is a rare mesenchymal neoplasm that affects deep soft tissues.

- Liposarcomas are often found in a significantly advanced condition due to the absence of distinctive symptoms.

- Surgery represents the elective therapy for liposarcomas, but the tendency to relapse, especially locally, is high.

- Eribulin represents a novel therapeutic tool for patients affected by advanced liposarcoma.

- We report the case of a 57-year-old male subject with a medical history of primary idiopathic neutropenia.

- The patient was affected by retroperitoneal advanced, anthracycline-pretreated liposarcoma.

- The patient experienced a long, beneficial clinical effect following eribulin treatment.

- Eribulin was demonstrated to be safe, even in presence of a personal history of idiopathic neutropenia.

\section{Author contributions}

All authors were involved in the writing and revision of this manuscript and have provided final approval to submit.

\section{Financial \& competing interests disclosure}

The authors have no relevant affiliations or financial involvements with any organization or entity with a financial interest in or a financial conflict with the subject matter or materials discussed in the manuscript. This includes employment, consultancies, honoraria, stock ownership or options, expert testimony, grants or patents received or pending, or royalties.

Medical writing support was provided by A Forte on behalf of Content Ed Net, and funded by Eisai.

\section{Ethical conduct of research}

The authors state that they have followed the principles outlined in the Declaration of Helsinki for all human or animal experimental investigations. In addition, for investigations involving human subjects, informed consent has been obtained from the participants involved.

\section{References}

Papers of special note have been highlighted as: • of interest; $\bullet \bullet$ of considerable interest

1. Lin ZC, Chang XZ, Huang XF et al. Giant liposarcoma of the esophagus: a case report. World J. Gastroenterol. 21(33), 9827-9832 (2015).

2. Crago AM, Brennan MF. Principles in management of soft tissue sarcoma. Adv. Surg. 49, 107-122 (2015).

3. Conyers R, Young S, Thomas DM. Liposarcoma: molecular genetics and therapeutics. Sarcoma 2011, 483154 (2011).

4. Thway K. Well-differentiated liposarcoma and dedifferentiated liposarcoma: an updated review. Semin. Diagn. Pathol. 36(2), 112-121 (2019).

5. Mansfield SA, Pollock RE, Grignol VP. Surgery for abdominal well-differentiated liposarcoma. Curr. Treat. Options Oncol. 19(1), 1 (2018). 
6. Dei Tos AP. Liposarcomas: diagnostic pitfalls and new insights. Histopathology 64(1), 38-52 (2014).

7. Katz D, Palmerini E, Pollack SM. More than 50 subtypes of soft tissue sarcoma: paving the path for histology-driven treatments. Am. Soc. Clin. Oncol. Educ. Book 38, 925-938 (2018).

8. Crago AM, Dickson MA. Liposarcoma: multimodality management and future targeted therapies. Surg. Oncol. Clin. N. Am. 25(4), 761-773 (2016).

9. Ducoulombier A, Cousin S, Kotecki N et al. Gemcitabine-based chemotherapy in sarcomas: a systematic review of published trials. Crit. Rev. Oncol. Hematol. 98, 73-80 (2016).

10. Judson I, Verweij J, Gelderblom H et al. Doxorubicin alone versus intensified doxorubicin plus ifosfamide for first-line treatment of advanced or metastatic soft-tissue sarcoma: a randomised controlled Phase III trial. Lancet Oncol. 15(4), 415-423 (2014).

- An assessment of potentiated doxorubicin + ifosfamide dosing on survival of patients with advanced soft-tissue sarcoma compared with doxorubicin alone.

11. Noujaim J, Thway K, Sheri A et al. Histology-driven therapy: the importance of diagnostic accuracy in guiding systemic therapy of soft tissue tumors. Int. J. Surg. Pathol. 24(1), 5-15 (2016).

12. Bai RL, Paull KD, Herald CL et al. Halichondrin B and homohalichondrin B, marine natural products binding in the vinca domain of tubulin. Discovery of tubulin-based mechanism of action by analysis of differential cytotoxicity data. J. Biol. Chem. 266(24), 15882-15889 (1991).

13. Kuznetsov G, Towle MJ, Cheng $\mathrm{H}$ et al. Induction of morphological and biochemical apoptosis following prolonged mitotic blockage by halichondrin B macrocyclic ketone analog E7389. Cancer Res. 64(16), 5760-5766 (2004).

14. Funahashi $\mathrm{Y}$, Okamoto $\mathrm{K}$, Adachi $\mathrm{Y}$ et al. Eribulin mesylate reduces tumor microenvironment abnormality by vascular remodeling in preclinical human breast cancer models. Cancer Sci. 105(10), 1334-1342 (2014).

15. Schoffski P, Ray-Coquard IL, Cioffi A et al. Activity of eribulin mesylate in patients with soft-tissue sarcoma: a Phase II study in four independent histological subtypes. Lancet Oncol. 12(11), 1045-1052 (2011).

-. An assessment of the activity and safety of eribulin in four strata of patients with different types of soft-tissue sarcoma.

16. Cortes J, O'Shaughnessy J, Loesch D et al. Eribulin monotherapy versus treatment of physician's choice in patients with metastatic breast cancer (EMBRACE): a Phase III open-label randomised study. Lancet 377(9769), 914-923 (2011).

17. Thara E, Gitlitz BJ. Eribulin: a new-generation antimicrotubule agent in lung cancer therapy. Future Oncol. 10(12), 1913-1924 (2014).

18. Schöffski P, Chawla S, Maki RG et al. Eribulin versus dacarbazine in previously treated patients with advanced liposarcoma or leiomyosarcoma: a randomised, open-label, multicentre, Phase III trial. Lancet 387(10028), 1629-1637 (2016).

-. Phase III trial demonstrating a significant advantage in terms of survival with eribulin in patients with liposarcoma and leiomyosarcoma. 